Research Article

\title{
The Oscillatory of Linear Conformable Fractional Differential Equations of Kamenev Type
}

\author{
Hui Liu and Run Xu \\ School of Mathematical Sciences, Qufu Normal University, Qufu 273165, Shandong, China \\ Correspondence should be addressed to Run Xu; xurun_2005@163.com
}

Received 14 January 2020; Accepted 10 March 2020; Published 9 April 2020

Academic Editor: Francisco R. Villatoro

Copyright (c) 2020 Hui Liu and Run Xu. This is an open access article distributed under the Creative Commons Attribution License, which permits unrestricted use, distribution, and reproduction in any medium, provided the original work is properly cited.

In this paper, the oscillatory of the Kamenev-type linear conformable fractional differential equations in the form of $\left(p(t) y^{(\alpha+1)}(t)\right)^{(\alpha)}+y^{(\alpha+1)}(t)+q(t) y(t)=0$ is studied, where $t \geq t_{0}$ and $0<\alpha \leq 1$. By employing a generalized Riccati transformation technique and integral average method, we obtain some oscillation criteria for the equation. We also give some examples to illustrate the significance of our results.

\section{Introduction}

The fractional derivative originated in the 17 th century and formed a relatively complete form in the 19th century. In the existing literature, we can find many definitions of the fractional integral and fractional differential given by these scholars, such as Riemann-Liouville, Caputo, Weyl, Hadamard, and Chen. In the past decades, fractional differential equations have been widely used in viscoelasticity, electrical networks, signal processing, systems identification, and other natural phenomena and physical problems, fluid flow, rheology, and many other fields. There are many literature studies that have investigated the existence, uniqueness, and stability of fractional differential equations and a series of conclusions about the qualitative of solutions, see [1-9] and their references.

In 2014, a new definition, which is called the conformable derivative, was proposed in the literature [10], and the properties and calculations of conformable derivatives have been studied in the literature [11-14]. It almost satisfied all the properties of the normal derivative: the derivative of constant is zero, the rules for the product and quotient of two functions, and the chain rule. Because it combines the best properties, many research studies have increased their research on it in recent years. Although there is much about the oscillations of fractional differential equations (see
[15-28] and the references therein), there is little about the conformable fractional differential equations of Kamenev type.

In 1978, Kamenev [7] studied the oscillation of the following:

$$
\left(p(t) y^{(\alpha)}(t)\right)^{(\alpha)}+q(t) y(t)=0, \quad t \geq t_{0}
$$

where $p \in C\left(\left[t_{0}, \infty\right),(0, \infty)\right), q \in C\left(\left[t_{0}, \infty\right), R\right), 0<\alpha \leq 1$ and $q$ might change signs. And the above equation is oscillation under the condition

$$
\lim \sup \frac{1}{t^{m}} \int_{t_{0}}^{t}(t-s)^{m} q(s) \mathrm{d} s=\infty, \quad m>1 .
$$

In 2019, Shao and Zhaowen [14] established new oscillation criteria of Kamenev type for linear conformable of the above equation, where $p \in C\left(\left[t_{0}, \infty\right),(0, \infty)\right), q \in C$ $\left(\left[t_{0}, \infty\right), R\right), 0<\alpha \leq 1$ and $q$ might change signs.

Inspired by the above, by extending the order of the equation containing conformable fractional derivative and increasing the number of terms, we make it have new application value.

In this paper, we discuss the oscillatory behavior about linear conformable fractional differential equations of Kamenev type of the following: 


$$
\left(p(t) y^{(\alpha+1)}(t)\right)^{(\alpha)}+y^{(\alpha+1)}(t)+q(t) y(t)=0, \quad t \geq t_{0},
$$

where $p \in C\left(\left[t_{0}, \infty\right),(0, \infty)\right), q \in C\left(\left[t_{0}, \infty\right), R\right), 0<\alpha \leq 1$.

In this paper, we always assume that the following conditions are in force:

$\left(\mathrm{C}_{1}\right)$ : there exist a constant $k \in N^{+}$such that $\left(y^{(\alpha)}(t) / y^{(\alpha+1)}(t)\right) \geq k, t \geq t_{0}$.

$\left(\mathrm{C}_{2}\right): H(t, s)$ is a continuous function and satisfies the following:

(1) $H(t, t)=0, H(t, s)>0$ for $t>s \geq t_{0}$.

(2) $H$ has a continuous and nonpositive partial derivative on $D_{0}$ with respect to the second variable, where $D_{0}=\left\{(t, s): t>s \geq t_{0}\right\}$.

(3) Let $h: D_{0} \longrightarrow R^{+}$be a continuous function and satisfy

$$
H_{s}(t, s)=-h(t, s) \sqrt{H(t, s)} .
$$

\section{Main Results}

First, we present the definition and properties of conformable fractional derivatives and integral.

$\left(D_{1}\right)$ : the left conformable fractional derivative starting from $t_{0}$ of a function $f:\left[t_{0}, \infty\right) \longrightarrow R$ of order $0<\alpha \leq 1$ is defined by

$$
\left(T_{t_{0}}^{\alpha} f\right)(t)=f^{(\alpha)}(t)=\lim _{\epsilon \longrightarrow 0} \frac{f\left(t+\epsilon\left(t-t_{0}\right)^{1-\alpha}\right)-f(t)}{\epsilon},
$$

where $t_{0}=0$, we write $T_{\alpha}$. If $\left(T_{t_{0}}^{\alpha} f\right)(t)$ exists on $\left(t_{0}, t_{1}\right)$, then

$$
\left(T_{t_{0}}^{\alpha} f\right)\left(t_{0}\right)=\lim _{t \longrightarrow t_{0}^{+}} f^{(\alpha)}(t) .
$$

If $f$ is differentiable, then

$$
\left(T_{t_{0}}^{\alpha} f\right)(t)=f^{(\alpha)}(t)=\left(t-t_{0}\right)^{1-\alpha} f^{\prime}(t) .
$$

$\left(\mathrm{D}_{2}\right)$ : if functions $f, g \in C[(0, \infty), R]$ are $\alpha$-differential for $t_{0}>0, \alpha \in(0,1]$ and continuous at $t_{0}$.

(1) For all real constants $a$ and $b$, we can get $T_{t_{0}}^{\alpha}(a f+b g)=a T_{t_{0}}^{\alpha}(f)+b T_{t_{0}}^{\alpha}(g)$

(2) $T_{t_{0}}^{\alpha}(f g)=f T_{t_{0}}^{\alpha}(g)+g T_{t_{0}}^{\alpha}(f)$

(3) For all $p, T_{t_{0}}^{\alpha}\left(t^{p}\right)=p t^{p-\alpha}$

(4) $T_{t_{0}}^{\alpha}(f / g)=\left(g T_{t_{0}}^{\alpha}(f)-f T_{t_{0}}^{\alpha}(g) / g^{2}\right)$, for $g \neq 0$

(5) When $c$ is a constant, we have $T_{t_{0}}^{\alpha}(c)=0$

$\left(\mathrm{D}_{3}\right):$ let $f, g:\left[t_{0}, t_{1}\right] \longrightarrow R$ be two functions, and $f g$ is differentiable. Then,

$\int_{t_{0}}^{t_{1}} f(s) g^{(\alpha)}(s) \mathrm{d}_{t_{0}}^{\alpha} s=\left.f(s) g(s)\right|_{t_{0}} ^{t_{1}}-\int_{t_{0}}^{t_{1}} g(s) f^{(\alpha)}(s) \mathrm{d}_{t_{0}}^{\alpha} s$.

Theorem 1. Suppose that $\left(C_{1}\right)$ and $\left(C_{2}\right)$ hold, and there exists a function $r(t) \in C\left(\left[t_{0}, \infty\right), R^{+}\right)$such that

$$
\limsup _{\mathrm{t} \longrightarrow \infty} \frac{1}{H\left(t, t_{0}\right)} \int_{t_{0}+1}^{t} H(t, s)\left[r(s) q(s)-\frac{1}{64 k} \frac{z^{2}(s)}{H(t, s)}\right] \mathrm{d}_{t_{0}}^{\alpha} s=\infty,
$$

then equation (3) is oscillatory, where $M_{+}(s)=\max \{0$, $\left.r^{(\alpha)}(s) p(s)-r(s)\right\}$,

$$
Z(s)=(r(s) p(s))^{-(3 / 2)} \frac{M_{+}(s) H(t, s)}{\left(s-t_{0}\right)^{1-\alpha} h(t, s)} .
$$

Proof. On the contrary, we suppose that equation (3) has a nonoscillation solution $y(t) \neq 0, t \geq t_{0}$. Defining $V(t)$ as a generalized Riccati substitution,

$$
V(t)=r(t) \frac{p(t) y^{(\alpha+1)}(t)}{y(t)} .
$$

From $\left(\mathrm{C}_{1}\right),(3)$, and $\left(\mathrm{D}_{2}\right)$, we obtain

$$
\begin{aligned}
V^{(\alpha)}(t) & =r^{(\alpha)}(t) \frac{p(t) y^{(\alpha+1)}(t)}{y(t)}+r(t)\left[\frac{p(t) y^{(\alpha+1)}(t)}{y(t)}\right]^{(\alpha)} \\
& =\frac{r^{(\alpha)}(t)}{r(t)} V(t)+r(t) \frac{\left.\left[p(t) t y^{(\alpha+1)} n(t)\right)\right]^{(\alpha)} y(t)-y^{(\alpha)}(t) p(t) y^{(\alpha+1)}(t)}{y^{2}(t)} \\
& =\frac{r^{(\alpha)}(t)}{r(t)} V(t)-r(t) q(t)-r(t) \frac{y^{(\alpha+1)}(t)}{y(t)}-r(t) \frac{p(t) y^{(\alpha)}(t) y^{(\alpha+1)}(t)}{y^{2}(t)} \\
& =\frac{r^{(\alpha)}(t)}{r(t)} V(t)-r(t) q(t)-\frac{V(t)}{p(t)}-\frac{V^{2}(t)}{r(t) p(t)} \cdot \frac{y^{(\alpha)}(t)}{y^{(\alpha+1)}(t)} \\
& \leq \frac{r^{(\alpha)}(t) p(t)-r(t)}{r(t) p(t)} V(t)-\frac{k}{r(t) p(t)} V^{2}(t)-r(t) q(t) \\
& \leq \frac{M_{+}(t)}{r(t) p(t)} V(t)-\frac{k}{r(t) p(t)} V^{2}(t)-r(t) q(t),
\end{aligned}
$$


that is,

$$
V^{(\alpha)}(t) \leq \frac{M_{+}(t)}{r(t) p(t)} V(t)-\frac{k}{r(t) p(t)} V^{2}(t)-r(t) q(t) .
$$

Multiply both sides of (13) by $H(t, s)$, and integrate from $T$ to $t_{0}\left(T \geq t_{0} \geq t_{0}\right)$. In accordance with $\left(\mathrm{D}_{1}\right),\left(\mathrm{C}_{2}\right),(13)$, and the integration by parts, we get

$$
\begin{aligned}
\int_{T}^{t} H(t, s) r(s) q(s) \mathrm{d}_{t_{0}}^{\alpha} s \leq & -\int_{T}^{t} H(t, s) V^{(\alpha)}(s) \mathrm{d}_{t_{0}}^{\alpha} s+\int_{T}^{t} H(t, s) \frac{M_{+}(s)}{r(s) p(s)} V(s) \mathrm{d}_{t_{0}}^{\alpha} s \\
& -\int_{T}^{t} H(t, s) \frac{k}{r(s) p(s)} V^{2}(s) \mathrm{d}_{t_{0}}^{\alpha} s=H(t, s) v(T)-\int_{T}^{t} v(s) H^{(\alpha)}(t, s) \mathrm{d}_{t_{0}}^{\alpha} s \\
& +\int_{T}^{t} H(t, s) \frac{M_{+}(s)}{r(s) p(s)} V(s) \mathrm{d}_{t_{0}}^{\alpha} s-\int_{T}^{t} H(t, s) \frac{k}{r(s) p(s)} V^{2}(s) \mathrm{d}_{t_{0}}^{\alpha} s \\
= & H(t, s) V(T)-\int_{T}^{t}\left(s-t_{0}\right)^{1-\alpha} h(t, s) \sqrt{H(t, s)} V(s) \mathrm{d}_{t_{0}}^{\alpha} s+\int_{T}^{t} H(t, s) \frac{M_{+}(s)}{r(s) p(s)} V(s) \mathrm{d}_{t_{0}}^{\alpha} s \\
& -\int_{T}^{t} H(t, s) \frac{k}{r(s) p(s)} V^{2}(s) \mathrm{d}_{t_{0}}^{\alpha} s \\
= & H(t, T) V(T) \\
& -\int_{T}^{t}\left\{H(t, s) \frac{k}{r(s) p(s)} V^{2}(s)+\left[\left(s-t_{0}\right)^{1-\alpha} h(t, s) \sqrt{H(t, s)}-H(t, s) \frac{M_{+}(s)}{r(s) p(s)}\right] V(s)\right\} \mathrm{d}_{t_{0}}^{\alpha} s .
\end{aligned}
$$

Let $\lambda=2, A=\sqrt{\left(s-t_{0}\right)^{1-\alpha} h(t, s)} \cdot[H(t, s)]^{1 / 4}$ and $B=$ $(1 / 2)\left(M_{+}(s) / r(s) p(s)\right) \cdot[H(t, s)]^{3 / 4} / \sqrt{\left(s-t_{0}\right)^{1-\alpha} h(t, s)}$.

From

$$
A^{\lambda}-\lambda A B^{\lambda-1} \geq(\lambda-1) B^{\lambda}, \quad \lambda>1
$$

$$
\begin{aligned}
\int_{T}^{t} H(t, s) r(s) q(s) \mathrm{d}_{t_{0}}^{\alpha} s \leq & H(t, T) V(T) \\
& -\int_{T}^{t}\left\{H(t, s) \frac{k}{r(s) p(s)} V^{2}(s)+\frac{1}{4} \frac{M_{+}^{2}(s)}{[r(s) p(s)]^{2}} H(t, s) \cdot \frac{\sqrt{H(t, s)}}{\left(s-t_{0}\right)^{1-\alpha} h(t, s)} V(s)\right\} \mathrm{d}_{t_{0}}^{\alpha} s \\
= & H(t, T) V(T) \\
& -\int_{T}^{t}\left[\sqrt{\left.\frac{k H(t, s)}{r(s) p(s)} V(s)+\frac{1}{8 \sqrt{k}} Z(s)\right]_{t_{0}}^{\alpha} s+\int_{T}^{t} \frac{1}{16 k} Z^{2}(S) \mathrm{d}_{t_{0}}^{\alpha} s}\right. \\
\leq & H(t, T) V(T)+\int_{T}^{t} \frac{1}{64 k} Z^{2}(S) \mathrm{d}_{t_{0}}^{\alpha} s,
\end{aligned}
$$

$$
\begin{aligned}
& \text { where } \quad Z(s)=(r(s) p(s))^{-(3 / 2)}\left(M_{+}(s) H(t, s)\right. \\
& \begin{array}{l}
\left.\left(s-t_{0}\right)^{1-\alpha} h(t, s)\right) \text {. } \\
\text { For } t \geq T \geq t_{0} \text {, we have } \\
\int_{T}^{t}\left[H(t, s) r(s) q(s)-\frac{1}{64 k} Z^{2}(S)\right] \mathrm{d}_{t_{0}}^{\alpha} s \leq H(t, T) V(T) \\
\quad \leq H(t, T)|V(T)| \leq H\left(t, t_{0}\right)|V(T)| .
\end{array}
\end{aligned}
$$

Letting $T=t_{0}+1$, we get

$$
\left.\int_{t_{0}+1}^{t}\left[H(t, s) r(s) q(s)-\frac{1}{64 k} Z^{2}(S)\right] \mathrm{d}_{t_{0}}^{\alpha} s \leq H\left(t, t_{0}\right) \mid V\left(t_{0}+1\right)\right) \mid \text {. }
$$

Dividing both sides of the above inequality by $H\left(t, t_{0}\right)$ and taking the upper limit in both sides as $t \longrightarrow \infty$, we have the contradict with (9). The proof is complete. 
Letting $m$ be an integer with $m>1$, then $H(t, t)=0, H(t, s)>0$. We have $h(t, s)=m(t-s)^{(m-2) / 2}$, so equation (9) is transformed to the following.

Corollary 1. If there exists a function $r(t) \in C\left(\left[t_{0}, \infty\right), R^{+}\right)$ and

$\limsup _{t \longrightarrow \infty} \frac{1}{t^{m}} \int_{t_{0}+1}^{t}(t-s)^{m}\left[r(s) q(s)-\frac{1}{64 k} \frac{J^{2}(s)}{H(t, s)}\right] \mathrm{d}_{t_{0}}^{\alpha} s=\infty$,

then equation (3) is oscillatory, where $J(s)=(r(s) p(s))^{-(3 / 2)}$ $\left(M_{+}(s)(t-s)^{(m+2) / 2} /\left(s-t_{0}\right)^{1-\alpha} m\right)$.

Before we introduce Theorem 2, we present the properties of the conformable fractional integral.

$\left(\mathrm{D}_{4}\right)$ : the left conformable fractional integral of order $0<\alpha \leq 1$ starting at $t_{0}$ is defined by

$$
\left(I_{t_{0}}^{\alpha} f\right)(t)=\int_{t_{0}}^{t}\left(s-t_{0}\right)^{\alpha-1} f(s) \mathrm{d} s=\int_{t_{0}}^{t} f(s) \mathrm{d}_{t_{0}}^{\alpha} s .
$$

We call the conformable fractional integral of a given function $f$ is $\alpha$-integrable if it exists.

$\left(\mathrm{D}_{5}\right)$ : assume that $f:[\alpha, \infty) \longrightarrow R$ is continuous and $0<\alpha \leq 1$. For all $t>t_{0}$, we can get

$$
\begin{aligned}
I_{t_{0}}^{\alpha} T_{t_{0}}^{\alpha}(f)(t) & =\int_{t_{0}}^{t}\left(s-t_{0}\right)^{\alpha-1}\left(s-t_{0}\right)^{1-\alpha} f^{\prime}(s) \mathrm{d} s \\
& =f(t)-f\left(t_{0}\right), \\
T_{t_{0}}^{\alpha} I_{t_{0}}^{\alpha}(f)(t) & =f(t) .
\end{aligned}
$$

$\left(D_{6}\right)$ (Cauchy-Schwarz inequality with conformable fractional derivative). Let $f, g:\left[t_{0}, \infty\right) \longrightarrow R$ be two functions such that $f^{2}$ and $g^{2}$ are $\alpha$-integrable. Then,

$$
\left|\int_{t_{0}}^{t} f(s) g^{(\alpha)}(s) \mathrm{d}_{t_{0}}^{\alpha} s\right|^{2} \leq \int_{t_{0}}^{t}|f(s)|^{2} \mathrm{~d}_{t_{0}}^{\alpha} s \cdot \int_{t_{0}}^{t}|g(s)|^{2} \mathrm{~d}_{t_{0}}^{\alpha} s .
$$

Theorem 2. Let $H(t, s)$ be defined as in $\left(C_{2}\right)$ and $\left(C_{1}\right)$ also hold. Suppose that

$$
\begin{gathered}
0<\inf _{s \geq t_{0}}\left[\liminf _{t \rightarrow \infty} \frac{H(t, s)}{H\left(t, t_{0}\right)}\right] \leq \infty, \\
\limsup _{t \rightarrow \infty} \frac{1}{H\left(t, t_{0}\right)} \int_{t_{0}}^{t} \frac{Z^{2}(s)}{16 k} \mathrm{~d}_{t_{0}}^{\alpha} s<\infty,
\end{gathered}
$$

hold. For every $T \geq t_{0}$, if there exists functions $S \in C\left(\left[t_{0}, \infty\right), R\right), r(t) \in C\left(\left[t_{0}, \infty\right), R^{+}\right)$such that

$$
\limsup _{t \rightarrow \infty} \frac{1}{H(t, s)} \int_{T}^{t}\left[H(t, s) r(s) q(s)-\frac{1}{64 k} Z(s)\right] \mathrm{d}_{t_{0}}^{\alpha} s \geq S(T),
$$

then equation (3) is oscillatory provided

$$
\int_{t_{0}+1}^{\infty} k \frac{S_{+}^{2}(s)}{r(s) p(s)} \mathrm{d}_{t_{0}}^{\alpha} s=\infty
$$

where $\quad r(t) \in C\left[t_{0}, \infty\right), \quad Z(s)=(r(s) p(s))^{-(3 / 2)}$ $\left(M_{+}(s) H(t, s) /\left(s-t_{0}\right)^{1-\alpha} h(t, s)\right)$, and $S_{+}(t)=\max \{S(t), 0\}$.

Proof. On the contrary, for all $t \geq t_{0}$, we suppose that equation (3) has a nonoscillation solution $y(t) \neq 0$. Define $V(t)$ as a generalized Riccati substitution:

$$
V(t)=r(t) \frac{p(t) y^{(\alpha+1)}(t)}{y(t)} .
$$

Proceeding as the proof of Theorem 1 , for $t \geq T \geq t_{0}$, from (16), we can get

$$
\begin{aligned}
& \int_{T}^{t}\left[H(t, s) r(s) q(s)-\frac{1}{64 k} Z(s)\right] \mathrm{d}_{t_{0}}^{\alpha} s \\
& \quad \leq H(t, T) V(T)-\int_{T}^{t}\left[\sqrt{\frac{k H(t, s)}{r(s) p(s)}} V(s)+\frac{1}{8 \sqrt{k}} Z(s)\right]^{2} \mathrm{~d}_{t_{0}}^{\alpha} s .
\end{aligned}
$$

Dividing both sides of the above inequality by $H(t, T)$, we obtain

$$
\begin{aligned}
& \frac{1}{H(t, T)} \int_{T}^{t}\left[H(t, s) r(s) q(s)-\frac{1}{64 k} Z(s)\right] \mathrm{d}_{t_{0}}^{\alpha} s \\
& \leq V(T)-\frac{1}{H(t, T)} \int_{T}^{t}\left[\sqrt{\frac{k H(t, s)}{r(s) p(s)}} V(s)+\frac{1}{8 \sqrt{k}} Z(s)\right]^{2} \mathrm{~d}_{t_{0}}^{\alpha} s .
\end{aligned}
$$

Immediately, it shows the following:

$$
\begin{aligned}
& \limsup _{t \rightarrow \infty} \frac{1}{H(t, s)} \int_{T}^{t}\left[H(t, s) r(s) q(s)-\frac{1}{64 k} Z(s)\right] \mathrm{d}_{t_{0}}^{\alpha} s \\
& \leq V(T)-\liminf _{t \longrightarrow \infty} \frac{1}{H(t, s)} \int_{T}^{t}\left[\sqrt{\frac{k H(t, s)}{r(s) p(s)}} V(s)+\frac{1}{8 \sqrt{k}} Z(s)\right]^{2} \mathrm{~d}_{t_{0}}^{\alpha} s .
\end{aligned}
$$

So, for all $T \geq t_{0}$, from (25), we have

$V(T) \geq S(T)+\liminf _{t \longrightarrow \infty} \frac{1}{H(t, s)} \int_{T}^{t}\left[\sqrt{\frac{k H(t, s)}{r(s) p(s)}} V(s)+\frac{1}{8 \sqrt{k}} Z(s)\right]^{2} \mathrm{~d}_{t_{0}}^{\alpha} s$.

It means that, for every $T \geq t_{0}$,

$V(T) \geq S(T)$

$\liminf _{t \rightarrow \infty} \frac{1}{H(t, s)} \int_{T}^{t}\left[\sqrt{\frac{k H(t, s)}{r(s) p(s)}} V(s)+\frac{1}{8 \sqrt{k}} Z(s)\right]^{2} \mathrm{~d}_{t_{0}}^{\alpha} s<\infty$.

Then, for every $T_{0}>t_{0}$, we obtain 


$$
\begin{gathered}
\infty>\liminf _{t \longrightarrow \infty} \frac{1}{H\left(t, T_{0}\right)} \int_{T_{0}}^{t}\left[\sqrt{\frac{k H(t, s)}{r(s) p(s)}} V(s)+\frac{1}{8 \sqrt{k}} Z(s)\right]^{2} \mathrm{~d}_{t_{0}}^{\alpha} s \\
\geq \liminf _{t \longrightarrow \infty}\left[\frac{1}{H\left(t, T_{0}\right)} \int_{T_{0}}^{t} \frac{k H(t, s)}{r(s) p(s)} V^{2}(s) \mathrm{d}_{t_{0}}^{\alpha} s\right. \\
\left.+\frac{1}{H\left(t, T_{0}\right)} \int_{T_{0}}^{t} \frac{1}{4} \sqrt{\frac{H(t, s)}{r(s) p(s)}} Z(s) V(s) \mathrm{d}_{t_{0}}^{\alpha} s\right] .
\end{gathered}
$$

For $t \geq T_{0}>t_{0}$, we let

$$
\begin{aligned}
& E(t)=\frac{1}{H\left(t, T_{0}\right)} \int_{T_{0}}^{t} \frac{k H(t, s)}{r(s) p(s)} V^{2}(s) \mathrm{d}_{t_{0}}^{\alpha} s, \\
& F(t)=\frac{1}{H\left(t, T_{0}\right)} \int_{T_{0}}^{t} \frac{1}{4} \sqrt{\frac{H(t, s)}{r(s) p(s)}} Z(s) V(s) \mathrm{d}_{t_{0}}^{\alpha} s .
\end{aligned}
$$

From (3), we have

$$
\liminf _{t \longrightarrow \infty}[F(t)+E(t)]<\infty .
$$

Next, we claim that

$$
\int_{T_{0}}^{\infty} k \cdot \frac{V^{2}(s)}{r(s) p(s)} \mathrm{d}_{t_{0}}^{\alpha} s<\infty .
$$

From (23), we can find a constant $\beta$ that will allow

$$
\inf _{s \geq t_{0}}\left[\liminf _{t \longrightarrow \infty} \frac{H(t, s)}{H\left(t, t_{0}\right)}\right]>\beta>0 .
$$

If (36) is not true, then for every $\tau>0$, we can find $T_{1}>T_{0}$ such that

$$
\int_{T_{0}}^{t} k \cdot \frac{V^{2}(s)}{r(s) p(s)} \mathrm{d}_{t_{0}}^{\alpha} s \geq \frac{2 \tau}{\beta}, \quad t>T_{1} .
$$

From $\left(D_{3}\right)$, we obtain the following:

$$
\begin{aligned}
E(t) & =\frac{1}{H\left(t, T_{0}\right)} \int_{T_{0}}^{t} H(t, s)\left[\int_{T_{0}}^{s} k \cdot \frac{V^{2}(\xi)}{r(\xi) p(\xi)} \mathrm{d}_{t_{0}}^{\alpha} \xi\right]^{(\alpha)} \mathrm{d}_{t_{0}}^{\alpha} s \\
& =-\frac{1}{H\left(t, T_{0}\right)} \int_{T_{0}}^{t}\left[\int_{T_{0}}^{s} k \cdot \frac{V^{2}(\xi)}{r(\xi) p(\xi)} \mathrm{d}_{t_{0}}^{\alpha} \xi\right][H(t, s)]_{s}^{(\alpha)} \mathrm{d}_{t_{0}}^{\alpha} s \\
& \geq-\frac{1}{H\left(t, T_{0}\right)} \int_{T_{1}}^{t}\left[\int_{T_{0}}^{s} k \cdot \frac{V^{2}(\xi)}{r(\xi) p(\xi)} \mathrm{d}_{t_{0}}^{\alpha} \xi\right][H(t, s)]_{s}^{(\alpha)} \mathrm{d}_{t_{0}}^{\alpha} s \\
& \geq-\frac{2 \tau}{\beta} \frac{1}{H\left(t, T_{0}\right)} \int_{T_{1}}^{t}[H(t, s)]_{s}^{(\alpha)} \mathrm{d}_{t_{0}}^{\alpha} s \\
& \geq \frac{2 \tau}{\beta} \frac{H\left(t, T_{1}\right)}{H\left(t, T_{0}\right)}, \quad t \geq T_{1}>T_{0} .
\end{aligned}
$$

Thus, (4) and (7) give $[H(t, s)]_{s}^{(\alpha)} \leq 0$, for $t \geq T_{1}>T_{0}$, and we have $H\left(t, T_{0}\right) \geq H\left(t, T_{1}\right)>0$.

However,

$$
\liminf _{t \rightarrow \infty} \frac{H\left(t, T_{1}\right)}{H\left(t, T_{0}\right)}>\beta, \quad T_{1}>T_{0} .
$$

Noticing that $T_{0}>t_{0}$ can be arbitrarily close to $t_{0}$ and $H$ is continuous, we choose $T_{2} \geq T_{1}$ such that, for $t \geq T_{2}$,

$$
\frac{H\left(t, T_{1}\right)}{H\left(t, T_{0}\right)} \geq \frac{\beta}{2} .
$$

So, from (39) and (41), we have

$$
E(t) \geq \tau, \quad t \geq T_{2},
$$

and $\tau>0$ is arbitrary, and we obtain

$$
\lim _{t \longrightarrow \infty} E(t)=\infty \text {. }
$$

In the interval $\left[T_{0}, \infty\right)$, we select a sequence $t_{i}$, $i=1,2, \ldots$, which makes $\lim _{i \rightarrow \infty} t_{i}=\infty$, and

$$
\lim _{i \longrightarrow \infty}\left[E\left(t_{i}\right)+F\left(t_{i}\right)\right]=\liminf _{t \longrightarrow \infty}[E(t)+F(t)] .
$$

From (35), we can always find a constant $M$ which lets

$$
E\left(t_{i}\right)+F\left(t_{i}\right) \leq M, \quad i=1,2, \ldots
$$

Through (43) and (45), we gain

$$
\begin{aligned}
& \lim _{i \longrightarrow \infty} E\left(t_{i}\right)=\infty, \\
& \lim _{i \longrightarrow \infty} F\left(t_{i}\right)=-\infty .
\end{aligned}
$$

The above also show that

$$
\lim _{i \longrightarrow \infty} \frac{F^{2}\left(t_{i}\right)}{E\left(t_{i}\right)}=\infty .
$$

Using (46), divide both sides of (45) by $E\left(t_{i}\right)$, and because $i$ is sufficiently large, we receive

$$
1+\frac{F\left(t_{i}\right)}{E\left(t_{i}\right)} \leq \frac{M}{E\left(t_{i}\right)}<\frac{1}{2} .
$$

So, we can get

$$
\frac{F\left(t_{i}\right)}{E\left(t_{i}\right)}<-\frac{1}{2} .
$$

By $\left(D_{6}\right)$, and for every positive integer $i$, we gain

$$
\begin{aligned}
F^{2}\left(t_{i}\right)= & {\left[\frac{1}{H^{2}\left(t_{i}, T_{0}\right)} \int_{T_{0}}^{t_{i}} \frac{1}{4} \sqrt{\frac{H\left(t_{i}, s\right)}{r(s) p(s)}} Z(s) V(s) \mathrm{d}_{t_{0}}^{\alpha} s\right]^{2} } \\
\leq & {\left[\frac{1}{H\left(t_{i}, T_{0}\right)} \int_{T_{0}}^{t_{i}} \frac{1}{16 k} Z^{2}(s) \mathrm{d}_{t_{0}}^{\alpha} s\right] } \\
& \cdot\left[\frac{1}{H\left(t_{i}, T_{0}\right)} \int_{T_{0}}^{t_{i}} \frac{k H\left(t_{i}, s\right)}{r(s) p(s)} V^{2}(s) \mathrm{d}_{t_{0}}^{\alpha} s\right] \\
= & {\left[\frac{1}{H\left(t_{i}, T_{0}\right)} \int_{T_{0}}^{t_{i}} \frac{Z^{2}(s)}{16 k} \mathrm{~d}_{t_{0}}^{\alpha} s\right] E\left(t_{i}\right) . }
\end{aligned}
$$

That is, 


$$
\frac{F^{2}\left(t_{i}\right)}{E\left(t_{i}\right)} \leq \frac{1}{H\left(t_{i}, T_{0}\right)} \int_{T_{0}}^{t_{i}} \frac{Z^{2}(s)}{16 k} \mathrm{~d}_{t_{0}}^{\alpha} s .
$$

Using (37), we obtain

$$
\liminf _{t \rightarrow \infty} \frac{H\left(t, T_{0}\right)}{H\left(t, t_{0}\right)}>\beta
$$

Then, there exists $T_{0}^{\prime} \geq T_{0}$, for $t \geq T_{0}^{\prime}$ such that

$$
\frac{H\left(t, T_{0}\right)}{H\left(t, t_{0}\right)} \geq \beta
$$

So, we have for sufficiently large $i$,

$$
\frac{H\left(t_{i}, T_{0}\right)}{H\left(t_{i}, t_{0}\right)} \geq \beta
$$

For any large $i,(52)$ is going to be equal to

$$
\begin{aligned}
\frac{F^{2}\left(t_{i}\right)}{E\left(t_{i}\right)} & \leq \frac{1}{\beta} \frac{1}{H\left(t_{i}, t_{0}\right)} \int_{T_{0}}^{t_{i}} \frac{Z^{2}(s)}{16 k} \mathrm{~d}_{t_{0}}^{\alpha} s \\
& \leq \frac{1}{\beta} \frac{1}{H\left(t_{i}, t_{0}\right)} \int_{t_{0}}^{t_{i}} \frac{Z^{2}(s)}{16 k} \mathrm{~d}_{t_{0}}^{\alpha} s .
\end{aligned}
$$

Since $\lim _{i \longrightarrow \infty}\left(F^{2}\left(t_{i}\right) / E\left(t_{i}\right)\right)=\infty$, that implies

$$
\limsup _{t \rightarrow \infty} \frac{1}{H\left(t, t_{0}\right)} \int_{t_{0}}^{t} \frac{Z^{2}(s)}{16 k} \mathrm{~d}_{t_{0}}^{\alpha} s=\infty,
$$

which contradicts with (24). This gives (36), that is,

$$
\int_{t_{0}+1}^{\infty} k \cdot \frac{V^{2}(s)}{r(s) p(s)} \mathrm{d}_{t_{0}}^{\alpha} s<\infty,
$$

and by using $V(T) \geq S(T)>0$, we can gain

$$
\int_{t_{0}+1}^{\infty} k \cdot \frac{S_{+}^{2}(s)}{r(s) p(s)} \mathrm{d}_{t_{0}}^{\alpha} s \leq \int_{t_{0}+1}^{\infty} k \cdot \frac{V^{2}(s)}{r(s) p(s)} \mathrm{d}_{t_{0}}^{\alpha} s<\infty, \text { lim, }
$$

which contradicts with (26). The proof is complete.

Corollary 2. If there exists a function $r(t) \in C\left(\left[t_{0}, \infty\right), R^{+}\right)$ and a function $S \in C\left(\left[t_{0}, \infty\right), R\right), H(t, s)=(t-s)^{m}, m>1$. So, (24) and (25) are replaced by

$$
\begin{gathered}
\limsup _{t \longrightarrow \infty} \frac{1}{t^{m}} \int_{t_{0}}^{t} \frac{J^{2}(s)}{16 k} \mathrm{~d}_{t_{0}}^{\alpha} s<\infty, \\
\underset{t \rightarrow \infty}{\limsup } \frac{1}{t^{m}} \int_{T}^{t}\left[(t-s)^{m} r(s) q(s)-\frac{1}{64 k} J(s)\right] \mathrm{d}_{t_{0}}^{\alpha} s \geq S(T),
\end{gathered}
$$

where $\quad J(s)=(r(s) p(s))^{-(3 / 2)}\left(M_{+}(s)(t-s)^{(m+2) / 2} /\right.$ $\left.\left(s-t_{0}\right)^{1-\alpha} m\right)$; then, equation (3) is oscillatory.

\section{Example}

Consider the oscillation for the conformable fractional differential equations

$$
\left(y^{(\alpha+1)}(t)\right)^{(\alpha)}+y^{(\alpha+1)}(t)+y(t)=0 . t \geq t_{0}, \quad 0<\alpha \leq 1 .
$$

Here, $y^{(\alpha)}(t)$ is the conformable fractional derivative. Compared with (1.1), here $p(s)=1$, and let $r(s)=p(s)=1$, that is, $J(s)=0$. Using Corollary 1 , we can verify that

$$
\begin{aligned}
& \limsup _{t \rightarrow \infty} \frac{1}{t^{m}} \int_{t_{0}+1}^{t}(t-s)^{m}\left[r(s) q(s)-\frac{1}{64 k} \frac{J^{2}(s)}{H(t, s)}\right] \mathrm{d}_{t_{0}}^{\alpha} s \\
& \quad=\frac{1}{t^{m}} \int_{t_{0}+1}^{t}(t-s)^{m} \mathrm{~d}_{t_{0}}^{\alpha} s \\
& \quad=\frac{1}{t^{m}} \int_{t_{0}+1}^{t}(t-s)^{m}\left(s-t_{o}\right)^{\alpha-1} \mathrm{~d} s \\
& \geq \frac{1}{t^{m}} \int_{t_{0}+1}^{t}(t-s)^{m}\left(s-T_{1}\right)^{\alpha-1} \mathrm{~d} s .
\end{aligned}
$$

Letting $s-T_{1}=\xi$, we have

$$
\begin{aligned}
\int_{t_{0}+1}^{t} & (t-s)^{m}\left(s-T_{1}\right)^{\alpha-1} \mathrm{~d} s \\
= & \int_{0}^{t-T_{1}} \xi^{\alpha-1}\left[t-\left(\xi+T_{1}\right)\right]^{m} \mathrm{~d} \xi \\
= & \int_{0}^{t-T_{1}} \xi^{\alpha-1}\left[\left(t-T_{1}\right)-\xi\right]^{m} \mathrm{~d} \xi \\
= & \int_{0}^{t-T_{1}} \xi^{\alpha-1}\left(1-\frac{\xi}{t-T_{1}}\right)^{m}\left(t-T_{1}\right)^{m} \mathrm{~d} \xi \\
= & \left(t-T_{1}\right)^{m+1}\left(t-T_{1}\right)^{\alpha-1} \\
& \cdot \int_{0}^{t-T_{1}}\left(\frac{\xi}{t-T_{1}}\right)^{\alpha-1}\left(1-\frac{\xi}{t-T_{1}}\right)^{m} d\left(\frac{\xi}{t-T_{1}}\right) .
\end{aligned}
$$

Next, letting $v=\left(\xi / t-T_{1}\right)$, then the above one

$$
\begin{aligned}
= & \left(t-T_{1}\right)^{m+1}\left(t-T_{1}\right)^{\alpha-1} \\
& \cdot \int_{0}^{t-T_{1}}\left(\frac{\xi}{t-T_{1}}\right)^{\alpha-1}\left(1-\frac{\xi}{t-T_{1}}\right)^{m} d\left(\frac{\xi}{t-T_{1}}\right) \\
= & \left(t-T_{1}\right)^{m+\alpha} \int_{0}^{1} v^{\alpha-1}(1-v)^{m} \mathrm{~d} v .
\end{aligned}
$$

That is,

$$
\begin{aligned}
& \int_{t_{0}+1}^{t}(t-s)^{m}\left(s-T_{1}\right)^{\alpha-1} \mathrm{~d} s \\
& =(t-T)_{1}^{m+\alpha} B(m+1, \alpha),
\end{aligned}
$$

where $B(\cdot, \cdot)$ is the classical beta function defined by

$$
B(m, n)=\int_{0}^{1}(1-s)^{m-1} s^{n-1} \mathrm{~d} s .
$$


So, we have $\left(1 / t^{m}\right) \int_{t_{0}+1}^{t}(t-s)^{m} \mathrm{~d}_{t_{0}}^{\alpha} s=\left(1 / t^{m}\right)\left(t-T_{1}\right)^{m+\alpha}$ $B(m+1, \alpha)=\infty$, which shows (19) holds. From Corollary 1 , we have equation (3) is oscillatory.

\section{Conclusion}

In the paper, we concerned with the oscillation of the Kamenev type for linear conformable fractional differential equations by using generalized Riccati transformation, Cauchy-Schwarz inequality for the conformable fractional derivative, and average inequality technique. Because conformable derivative combines the best qualities of fractional derivatives, it is now well developed. We will continue to refine this part of the theory and continue to study the oscillation of fractional differential equations.

\section{Data Availability}

The authors declare that the data and material in the paper can be used publicly.

\section{Conflicts of Interest}

The authors declare that there are no conflicts of interest regarding the publication of this paper.

\section{Authors' Contributions}

HL carried out the main results and completed the corresponding proof. RX participated in the proof and helped to complete Section 3-Examples. All authors read and approved the final manuscript.

\section{Acknowledgments}

This research was supported by the National Science Foundation of China (11671227 and 11971015) and the Natural Science Foundation of Shandong Province (ZR2019MA034).

\section{References}

[1] K. S. Miller and B. Ross, An Introduce to the Fractional Calculus and Fractional Differential Equations, Wiley-Interscience, New York, NY, USA, 1993.

[2] G. Samko and A. A. Kilbas, Marichev, Fractional Integrals and Derivatives: Theory and Appdon Applications, Gordon \& Breach, Yverdon, Switzerland, 1993.

[3] I. Podlubny, "Fractional differential equations. An introduction to fractional derivatives, fraction differential equations, to methods of their solution and some of their applications," in Mathematics in Science and Engineering, Vol. 198, Academic Press, San Diego, CA, USA, 1999.

[4] A. A. Kilbas, H. M. Srivastava, and J. J. Trujillo, Theory and Applications of Fractional Differential Equations, Elsevier Science, Amsterdam, Netherlands, 2006.

[5] K. Diethelm, The Analysis of Fractional Differential Equations, Vol. 2004, Springer, Berlin, Germany, 2010.

[6] A. B. Malinowska, T. Odzijewicz, and D. F. M. Torres, Advanced Methods in the Fractional Calculus of Variations, Springer International Publishing, New York, NY, USA, 2015.
[7] I. V. Kamenev, "An integral test for conjugacy for second order linear differential equations," Akademiya Nauk SSSR Matematicheskie Zametki, vol. 23, no. 2, pp. 249-251, 1978.

[8] D. Baleanu and O. P. Agrawal, "Fractional Hamilton formalism within Caputo's derivative," Czechoslovak Journal of Physics, vol. 56, no. 10-11, pp. 1087-1092, 2006.

[9] T. Odzijewicz, A. B. Malinowska, and D. F. M. Torres, "Fractional variational calculus with classical and combined Caputo derivatives," Nonlinear Analysis: Theory, Methods \& Applications, vol. 75, no. 3, pp. 1507-1515, 2012.

[10] R. Khalil, M. Al Horani, A. Yousef, and M. Sababheh, "A new definition of fractional derivative," Journal of Computational and Applied Mathematics, vol. 264, pp. 65-70, 2014.

[11] T. Abdeljawad, "On conformable fractional calculus," Journal of Computational and Applied Mathematics, vol. 279, pp. 57-66, 2015.

[12] A. Atangana, D. Baleanu, and A. Alsaedi, "New properties of conformable derivative," Open Mathematics, vol. 13, no. 1, pp. 889-898, 2015.

[13] M. J. Lazo and D. F. M. Torres, "Variational calculus with conformable fractional derivatives," IEEE/CAA Journal of Automatica Sinica, vol. 4, no. 2, pp. 340-352, 2017.

[14] J. Shao and Z. Zhaowen, "Kamenev type oscillation criteria for linear conformable fractional differential equations," Discrete Dynamics in Nature and Society, vol. 2019, Article ID 2310185, 10 pages, 2019.

[15] Y. Sawano, H. T. Sobukawa, and H. Tanaka, "Limiting case of the boundedness of fractional integral operators on nonhomogeneous space," Journal of Inequalities and Applications, vol. 2006, no. 1, pp. 1-16, 2006.

[16] C. Daxue, "Oscillatory behavior of a class of fractional differential equations with damping," UPB Scientific Bulletin Series A, vol. 75, no. 1, 2013.

[17] R. Xu, "Oscillation criteria for nonlinear fractional differential equations," Journal of Applied Mathematics, vol. 2013, Article ID 971357, 7 pages, 2013.

[18] A. Akbulut, V. H. Hamzayev, and Z. V. Safarov, "Boundedness of rough fractional multilinear integral operators on generalized Morrey spaces," Journal of Inequalities and Applications, vol. 2015, no. 1, p. 234, 2015.

[19] Z. Bai and R. Xu, "The asymptotic behavior of solutions for a class of nonlinear fractional difference equations with damping term," Discrete Dynamics in Nature and Society, vol. 2018, Article ID 5232147, 11 pages, 2018.

[20] X. Wang and R. Xu, "The existence and uniqueness of solutions and lyapunov-type inequality for CFR fractional differential equations," Journal of Function Spaces, vol. 2018, Article ID 5875108, 7 pages, 2018.

[21] D. Zan and R. Xu, "The existence results of solutions for system of fractional differential equations with integral boundary conditions," Discrete Dynamics in Nature and Society, vol. 2018, Article ID 8534820, 8 pages, 2018.

[22] M. Wei, C. Jiang, and T. Li, "Oscillation of third-order neutral differential equations with damping and distributed delay," Advances in Difference Equations, vol. 2019, p. 426, 2019.

[23] H. Liu and R. Xu, "Oscillation for a class of right fractional differential equations on the right half line with damping," Discrete Dynamics in Nature and Society, vol. 2019, Article ID 4902718, 9 pages, 2019.

[24] O. Bazighifan, E. M. Elabbasy, and O. Moaaz, "Oscillation of higher-order differential equations with distributed delay," Journal of Inequalities and Applications, vol. 2019, p. 55, 2019. 
[25] O. Bazighifan and C. Cesarano, "Some new oscillation criteria for second-order neutral differential equations with delayed arguments," Mathematics, vol. 7, no. 7, p. 18, 2019.

[26] E. M. Elabbasy, C. Cesarano, O. Bazighifan, and O. Moaaz, "Asymptotic and oscillatory behavior of solutions of a class of higher order differential equation," Symmetry, vol. 11, no. 12, p. 1434, 2019.

[27] C. Cesarano and O. Bazighifan, "Oscillation of fourth-order functional differential equations with distributed delay," Axioms, vol. 8, no. 2, p. 61, 2019.

[28] C. Cesarano and O. Bazighifan, "Qualitative behavior of solutions of second order differential equations," Symmetry, vol. 11, no. 6, p. 777, 2019. 